\title{
Randomized clinical trial of open versus laparoscopic left lateral hepatic sectionectomy within an enhanced recovery after surgery programme (ORANGE II study)
}

\author{
E. M. Wong-Lun-Hing ${ }^{1,2}$, R. M. van Dam ${ }^{1,13}$, G. J. P. van Breukelen ${ }^{3,4}$, P. J. Tanis ${ }^{6}$, F. \\ Ratti $^{14}$, R. van Hillegersberg ${ }^{7}$, G. D. Slooter ${ }^{8}$, J. H. W. de Wilt ${ }^{9}$, M. S. L. Liem ${ }^{10}$, M. T. de \\ Boer $^{11}$, J. M. Klaase ${ }^{12}$, U. P. Neumann ${ }^{1,13}$, L. A. Aldrighetti ${ }^{14}$ and C. H. C. Dejong ${ }^{1,2,5,13}$, on \\ behalf of the ORANGE II Collaborative Group*
}

${ }^{1}$ Department of Surgery, ${ }^{2}$ Nutrim School for Nutrition and Translational Research in Metabolism, ${ }^{3}$ Department of Methodology and Statistics, ${ }^{4}$ CAPHRI School for Public Health and Primary Care, and ${ }^{5}$ GROW - School for Oncology and Developmental Biology, Maastricht University Medical Centre, Maastricht, and Departments of Surgery, ${ }^{6}$ Academic Medical Centre, Amsterdam, ${ }^{7}$ University Medical Centre Utrecht, Utrecht, ${ }^{8}$ Maxima Medical Centre, Veldhoven, ${ }^{9}$ Radboud University Medical Centre, Nijmegen, ${ }^{10}$ Deventer Hospital, Deventer, ${ }^{11}$ University Medical Centre Groningen, Groningen, and ${ }^{12}$ Medisch Spectrum Twente, Enschede, The Netherlands, ${ }^{13}$ Department of Surgery, University Hospital Aachen, Aachen, Germany, and ${ }^{14}$ Department of Surgery, San Raffaele Hospital, Milan, Italy

Correspondence to: Dr R. M. van Dam, Department of Surgery, Maastricht University Medical Centre, PO Box 5800, 6202 AZ Maastricht, The Netherlands (e-mail: r.van.dam@mumc.nl)

* Other members of the ORANGE II Collaborative Group are co-authors of this study and can be found under the heading Collaborators

Background: Laparoscopic left lateral sectionectomy (LLLS) has been associated with shorter hospital stay and reduced overall morbidity compared with open left lateral sectionectomy (OLLS). Strong evidence has not, however, been provided.

Methods: In this multicentre double-blind RCT, patients (aged 18-80 years with a BMI of $18-35 \mathrm{~kg} / \mathrm{m}^{2}$ and American Society of Anesthesiologists fitness grade of III or below) requiring left lateral sectionectomy (LLS) were assigned randomly to OLLS or LLLS within an enhanced recovery after 
surgery (ERAS) programme. All randomized patients, ward physicians and nurses were blinded to the procedure undertaken. A parallel prospective registry (open non-randomized (ONR) versus laparoscopic non-randomized (LNR)) was used to monitor patients who were not enrolled for randomization because of doctor or patient preference. The primary endpoint was time to functional recovery. Secondary endpoints were length of hospital stay (LOS), readmission rate, overall morbidity, composite endpoint of liver surgery-specific morbidity, mortality, and reasons for delay in discharge after functional recovery.

Results: Between January 2010 and July 2014, patients were recruited at ten centres. Of these, 24 patients were randomized at eight centres, and 67 patients from eight centres were included in the prospective registry. Owing to slow accrual, the trial was stopped on the advice of an independent Data and Safety Monitoring Board in the Netherlands. No significant difference in median (i.q.r.) time to functional recovery was observed between laparoscopic and open surgery in the randomized or non-randomized groups: 3.0 (3-5) days for OLLS versus 3.0 (3-3) days for LLLS; and 3.0 (3-3) days for ONR versus 3.0 (3-4) days for LNR. There were no significant differences with regard to LOS, morbidity, reoperation, readmission and mortality rates.

Conclusions: This RCT comparing open and laparoscopic LLS in an ERAS setting was not able to reach a conclusion on time to functional recovery, because it was stopped prematurely owing to slow accrual. Registration number: NCT00874224 (https://www.clinicaltrials.gov).

\section{+A: Introduction}

The left lateral segments of the liver can be resected by both open and laparoscopic approaches. The latter has become increasingly popular after the Louisville Statement of 2008, where experts concluded that laparoscopic left lateral sectionectomy (LLS) was a safe and effective approach for the management of surgical liver disease in the hands of experienced hepatobiliary and laparoscopic surgeons ${ }^{1}$. Later reviews, based mainly on retrospective data, showed favourable clinical outcomes after laparoscopic resection ${ }^{2,3}$. Studies specifically comparing open and laparoscopic LLS have hitherto been based on retrospective designs or with small sample size ${ }^{4,5}$. A meta-analysis of non-randomized studies showed a reduction in duration of operation, shorter overall length of hospital stay (LOS) and reduced morbidity after laparoscopic LLS ${ }^{6}$. This difference in LOS was, however, associated with significant heterogeneity among the included studies. 
Enhanced recovery after surgery (ERAS) programmes have been introduced in patients undergoing minor and major liver resections. Multiple studies ${ }^{7-15}$ have shown that these programmes are feasible, safe and effective in reducing median LOS in both open and laparoscopic resection. Data available at the time of design of this study also suggested that a further reduction in LOS after liver resection could be achieved when the observed delay between patient recovery and actual discharge was minimized, as reported for colonic resections ${ }^{10,16}$. Day of discharge from hospital is dependent on multiple factors, including patient expectations, local discharge logistics and cultural differences between countries, hospitals and surgeons. LOS may therefore be considered an inappropriate endpoint for comparison of surgical interventions. Within the ERAS programme for liver surgery, a composite endpoint has been defined: time to functional recovery. This endpoint, representing medical readiness for discharge, consisted of clear and objectively measurable criteria. A patient was considered functionally recovered if they had a normal or decreasing serum bilirubin level, good pain control with oral analgesia only, tolerance of solid food, no intravenous fluid support and independent mobility at the preoperative level ${ }^{10}$. Functionally recovered patients were generally capable of independently performing activities of daily living and were independent of hospital care.

The aim of this study was to compare open and laparoscopic liver surgery in a randomized, controlled, multicentre and blinded setting, in which all patients received a standardized liver resection within a standardized perioperative care programme, based on a standardized recovery outcome measure. A parallel registry to the randomized controlled arms was created to study an uninterrupted series for external validity. The hypothesis was that in patients undergoing laparoscopic LLS (LLLS), time to functional recovery would be reduced by 2 days compared with that in those having open LLS (OLLS).

\section{+A: Methods}

\section{$+B:$ Study design and participants}

This study (ORANGE II) was designed as a double-blind RCT with a parallel prospective registry of patients who could not be randomized owing to patient or surgeon preference. Patients were eligible for inclusion in the randomization if they required a liver LLS for accepted indications, if they were men or 
non-pregnant, non-lactating women aged $18-80$ years with a BMI of $18-35 \mathrm{~kg} / \mathrm{m}^{2}$ and an American Society of Anesthesiologists (ASA) grade of III or less. Exclusion criteria were: planned liver resection other than LLS, ASA grade above III, and underlying liver disease diagnosed before surgery.

Representatives of the ORANGE II study group from Maastricht University Medical Centre coordinated the trial and analysed the data. The study protocol was approved by medical ethics committees at each participating centre. Centres were approached by e-mail and could participate if laparoscopic and open liver surgery were performed on a routine basis and if an ERAS liver programme had been implemented. Fourteen European sites obtained ethical approval to enrol patients; twelve were located in the Netherlands, one in Germany and one in Italy. The study protocol was registered online at ClinicalTrials.gov (NCT00874224), and has been published ${ }^{17}$. The aim of the study was to compare LLLS with OLLS in terms of time to functional recovery (primary endpoint). The hypothesis was that time to functional recovery would be reduced by 2 days in patients undergoing LLLS.

\section{+B: Randomization and masking}

Patients were approached for participation in the outpatient clinic. All provided written informed consent before preoperative assessment. They received information and counselling related to the study intervention, ERAS programme and other study-related procedures. Patients were assigned randomly before admission in a $1: 1$ ratio to either OLLS or LLLS. Randomization was performed by each local study coordinator using a web-based system (TENALEA ${ }^{\circledR}$; FormsVision, Abcoude, The Netherlands) and block randomization. Randomization was stratified according to treatment, centre, sex and ASA grade. The allocated procedure was communicated to the operating surgeon(s). All randomized patients, ward physicians and nurses were blinded to the type of intervention by the use of a large fixed abdominal dressing until postoperative day (POD) 3.

Non-randomized patients were asked for permission to use their data. In doing so, they were assigned to the open non-randomized (ONR) or laparoscopic non-randomized (LNR) arm of the prospective registry, on the basis that this registry might increase the external validity of results obtained in the randomized study ${ }^{18,19}$. 


\section{+B: Procedures}

The intraoperative surgical technique was not standardized; surgeons in participating centres were free to use their preferred technique and devices to gain intra-abdominal access, perform hepatic parenchymal transection and maintain vascular control. Surgeons in each participating centre performed the allocated intervention based upon availability. Medical centres with liver surgeons early in the laparoscopic learning curve were assisted during the procedure by an experienced proctoring laparoscopic hepatopancreatobiliary (HPB) surgeon. Perioperative care for all patients in the study was standardized according to the ERAS programme and the perioperative care provided was based on daily guidelines (Table 1).

$+B$ : Outcomes

The primary outcome measure of this study for both the randomized and parallel cohorts was time to functional recovery. A patient was considered functionally recovered when all of the following criteria were fulfilled: adequate pain control with oral analgesia only; restoration of mobility to an independent or preoperative level; absence of intravenous fluid administration; ability to eat solid foods; and normal or decreasing serum bilirubin level or international normalized ratio (INR). The evaluation of time to functional recovery started on POD 0 and was scored until discharge from hospital using a standard checklist and patient diary. Patients were considered ready for discharge when the primary endpoint had been met, although it was up to the local logistics of each centre to define the actual moment of discharge. The delay between time to functional recovery and actual discharge was recorded and reasons for this delay were obtained.

Postoperative pain was registered twice daily using the validated, verbally administered, 11-point (0-10) numerical rating scale (NRS-11) ${ }^{20,21}$. Centres were free to provide either epidural or intravenous patient-controlled analgesia. No indwelling wound catheters were used in participating centres. Members of a specialized pain team asked patients to rate the intensity of their current pain on a scale from 0 (no pain) to 10 (worst possible pain). A score of 1-3 was considered to be mild, 4-6 moderate and 7-10 severe $^{22}$. 
To report the difference between preoperative and postoperative level of mobility, the ERAS Mobility Scale (EMS), derived from the Groningen Activity Restriction Scale ${ }^{23}$, was used. The EMS utilizes ten items of basic actions to compare the level of mobility before and after surgical intervention. When the patient reached the preoperative EMS level, or had a positive score for eight of ten items, they were considered independently mobile.

Fluid and solid food intake was monitored, and a normal tolerance was required before discharge. Tolerance was considered to be normal when oral intake solid food was resumed and continued for at least $24 \mathrm{~h}$.

At the time of design of the study it was decided to monitor the postoperative serum bilirubin concentration and INR to ensure that no patient was discharged with impaired liver function. Serum bilirubin levels and INR were measured before surgery and on POD 1 and 3.

Secondary outcomes were postoperative LOS, readmission rate, total morbidity according to the Clavien-Dindo classification ${ }^{24}$, composite endpoint of liver surgery-specific morbidity ${ }^{25}$, mortality, and reasons for delay in discharge after functional recovery.

$+B$ : Data collection and patient safety

Data were collected using both paper case report forms (CRFs) and an open-source clinical trial software platform (OpenClinica ${ }^{\circledR}$; Ikaza Research, Cambridge, Massachusetts, USA) in compliance with good clinical practice guidelines. The e-CRFs were stored in a secured database (Oracle Corporation, Redwood Shores, California, USA). A baseline assessment of mobility was performed on the day of admission. Venous blood samples were drawn before and after surgery, on POD 1 and 3. During admission, surgical details, data on time to functional recovery and complications were collected with a patient diary, a milestone checklist and standardized adverse event forms.

An independent Data and Safety Monitoring Board (DSMB) in the Netherlands evaluated the progress and quality of the trial and examined safety endpoints for each consecutive group of 25 patients. Baseline characteristics and serious adverse events were listed and presented in an unblinded fashion. 
Recommendations made by the DSMB were communicated to the medical ethics review committee of Maastricht University Medical Centre and all participating centres.

\section{+ B: Statistical analysis}

Time to functional recovery was used as the primary endpoint. Owing to lack of data on the reduction in time to functional recovery after liver surgery in an ERAS programme, a decision was made to use LOS for the purpose of power calculation, because this approached the primary endpoint most accurately. Based on a retrospective analysis of 31 patients in both ERAS and non-ERAS settings, who had undergone LLS from 1990 to 2010, the mean(s.d.) value for postoperative LOS after LLS in Maastricht University Medical Centre was 6.0(2.7) days. Thus a reduction in time to functional recovery of 2 days seemed feasible.

At 90 per cent power and $\alpha=0.05$ (two-tailed), a sample size of $2 \times 40$ patients in the randomization arms would be sufficient to detect this difference (two-tailed testing was planned to allow detection of an (unexpected) increase in time to functional recovery after LLLS compared with OLLS, and to be consistent with the two-sided confidence intervals to be reported). Assuming an expected withdrawal rate of 10 per cent or less, the participation of at least ten centres, and the required addition of one randomized patient per arm for every additional participating centre to compensate for the loss of degrees of freedom incurred in the data analysis (which takes centre and treatment $\times$ centre effects into account), a total sample size of 110 $(2 \times 55)$ was required. Patients were analysed according to the intention-to-treat principle, and analysis was performed with SPSS ${ }^{\circledR}$ software using Windows ${ }^{\circledR}$ version 21.0 (IBM, Armonk, New York, USA).

After each group of 25 included patients, a report was sent to the DSMB. An interim analysis was planned after randomization and completion of follow-up of 50 per cent of the total sample size. The DSMB provided the principal investigator with recommendations: no action needed, early stopping (due to clear benefit/harm of a treatment, futility, or new external evidence), extending recruitment or follow-up, stopping a single arm of the multi-arm trial, or sanctioning and/or proposing protocol changes.

Assumption of normality was checked using the Shapiro-Wilk test. Continuous numerical data were summarized by the median (i.q.r.) value per treatment arm. Analysis was performed with $\chi^{2}$ or Fisher's exact tests for binary outcomes, and Mann-Whitney $U$ or $t$ tests for continuous outcomes, depending on 
their normality. For the primary outcome time to functional recovery $\alpha=0.05$ (two-tailed) and for the secondary outcomes $\alpha=0.01$ (two-tailed) were used to correct for multiple testing.

Post hoc analyses to assess milestones during recovery of patients and compliance with elements of the ERAS protocol were also performed. To describe the compliance with individual ERAS elements per study group and centre, an overall compliance and the between-centre range was used. Per element, an 80 per cent cut-off value was set to qualify as compliant.

The outcome analyses were repeated with multiple linear regression to adjust for patient age, sex, ASA grade and centre effects. Differences between individual surgeons within the same centre with respect to time to functional recovery, LOS, and difference between time to functional recovery and LOS were also examined.

\section{+A: Results}

\section{$+B$ : Enrolment and randomization}

From January 2010 to 1 July 2014, ten of 14 centres that had ethical approval recruited patients for this study. A total of 104 patients were assessed for eligibility and 97 were included (Fig 1). Only 29 participants were randomized: 14 to OLLS and 15 to LLLS. The remaining 68 patients were included in the prospective registry. Some five patients were excluded after randomization: four required a larger hepatic resection and surgery was postponed in one woman because of pregnancy. One patient in the open arm of the prospective registry was also excluded after a preoperative change of procedure. No 30-day dropouts were observed in the RCT or prospective registry. A total of 91 patients (24 randomized and 67 prospective registry) were included in the intention-to-treat analysis.

The DSMB did not express any objections to continuation of the trial after the first two reports (October 2012 and April 2013). In the third report of January 2014, however, the DSMB expressed concerns about the ORANGE II study group, because of an accrual rate of 24 per cent in the RCT. In accordance with recommended criteria for accrual and scientific progress ${ }^{26}$, a trial should be closed if it is open for more than 24 months with an accrual rate of less than 25 per cent. Therefore, the DSMB advised 
continuation of the trial for a maximum of 6 months. Because enrolment remained slow, a decision was made by the investigators to stop the trial on 1 July 2014.

\section{$+B$ : Preference}

Sixty-seven (73.6 per cent) of the 91 included patients in this study had surgery based on preference for either the open or laparoscopic procedure, usually surgeon preference: nine of 13 patients (69 per cent) in the open arm and 39 of 54 (72 per cent) of those in the laparoscopic arm of the prospective registry.

\section{+B: Patient demographics and surgical outcome}

Baseline patient characteristics (Table 2) and surgical outcomes (Table 3) were distributed equally between the groups in the randomized part study (OLLS versus LLLS), as well as in the prospective registry (ONR versus LNR). There appeared to be more patients with a history of previous abdominal surgery in the LNR group and a longer median duration of surgery in the LLLS group, but after correction for multiple testing these differences were not significant.

\section{+B: Primary outcome}

Time to functional recovery did not significantly differ between OLLS and LLLS groups, or between ONR and LNR groups (Table 4). In the OLLS group, the median time to functional recovery was 3 (3-5) days, compared with $3(3-3)$ days in the LLLS group $(P=0.284)$. The same median time to functional recovery was found in the registry groups: 3 (3-3) days for ONR versus 3 (3-4) days for LNR $(P=0.529)$. Subanalysis of individual functional recovery criteria revealed quicker tolerance of solid food in favour of the LNR group (2 (1-2) days versus 1 (1-1) day following ONR; $P=0.002)$.

\section{$+B$ : Secondary outcomes}

Median LOS did not differ significantly following OLLS compared with LLLS: 4.5 (4-6) versus 4 (3-5) days respectively ( $P=0.049$ ) (note that $\alpha=0.01$ for secondary outcomes in view of multiple testing). Median LOS in the prospective registry was also comparable: 5 (4-7) days for ONT versus 4 (3-5) days for LNR $(P=0.064)$. The reasons for delayed discharge after functional recovery per group are shown in Table 4. Overall 39, 18 and 10 per cent of the delay was logistical or medical in nature, or based on patient request, respectively. 
Overall morbidity was the same for open and laparoscopic procedures, with no significant differences between Clavien-Dindo complication severity grades ${ }^{23}$ or the composite endpoint for liver surgery-related morbidity (Table 5).

A total of five patients developed major morbidity (grade III or above). One patient in the open arm of the RCT died after developing a pulmonary embolism and cardiac arrest. One randomized patient developed a wound infection after LLLS, which was re-explored under local anaesthesia. In the open arm of the registry, one patient required surgery after a postoperative intra-abdominal bleed with haemodynamic instability. In the laparoscopic arm of the registry, one patient was admitted to the ICU for postresectional liver failure, and one was readmitted and received percutaneous radiological drainage after developing an intra-abdominal abscess.

$+B$ : Compliance with the ERAS protocol

No group was fully compliant with all protocol items. The OLLS, LLLS, ONR and LNR groups were compliant (more than 80 per cent) with between 10 and 14 of the 22 ERAS elements (Table 6). Postoperative epidural analgesia was provided to 82, 69, 77 and 65 of patients in the OLLS, LLLS, ONR and LNR groups respectively. No data were available to score the elements ileus prevention (laxatives) and provision of oral analgesia on POD 1.

$+B$ : Regression analyses

Repeating the outcome analyses with multiple linear regression to adjust for patient age, sex, ASA grade and centre effects essentially confirmed the results of the Mann-Whitney $U$ tests. However, analysis of residuals showed a clear outlier in both the open surgery arm of the RCT and the registry with respect to the outcome LOS. Therefore, all analyses, non-parametric as well as regression, were repeated. Without those outliers, the new results increased both $P$ values for LOS to greater than 0.100 . Finally, owing to the very small sample size, differences between surgeons within the same centre could not be evaluated.

\section{+A: Discussion}

This RCT comparing open and laparoscopic LLS in an ERAS setting was not able to reach a meaningful conclusion on time to functional recovery because it had to be stopped prematurely owing to poor 
recruitment. No difference in time to functional recovery was found after LLLS compared with the OLLS in the randomized trial, but this analysis is underpowered. A prospective registry of all patients not randomized due to surgeon or patient preference also failed to show a difference in functional recovery. Considering the secondary endpoints, no differences between surgical procedures were observed with regard to blood loss, duration of surgery, LOS, morbidity, reoperation, readmissions and mortality rates.

The main contributor to poor recruitment was individual surgeons’ preference for the laparoscopic procedure. This lead to a preference : randomization ratio of almost $3: 1$. Clinical equipoise was assumed at the time of design and start of this study. Based on a worldwide survey among HPB surgeons, an RCT and prospective register comparing open and laparoscopic techniques was considered necessary ${ }^{27}$. The majority of participating centres in the study had indicated that they considered a trial to be feasible and were willing to randomize patients. It was on this basis that it was deemed not necessary to perform a feasibility study. It is clear, however, that clinical equipoise was no longer present during the recruitment period. A recent expert statement from the International Consensus Conference for Laparoscopic Liver Resection held at Morioka, Japan, in 2014 stated that minor laparoscopic liver resection, including LLS, has become standard practice ${ }^{28}$, and this evolving change in surgical attitudes is likely to have influenced the present study.

The slow accrual might also be related to the lower incidence of LLS than an anticipated 10 per cent of the total volume of liver resections. Replacement of LLS by parenchyma-saving strategies using metastasectomy or local ablation procedures may also have been a contributing factor to slow recruitment.

Despite these limitations, the study has several strengths. No RCT has compared open and laparoscopic liver surgery. Other studies comparing open and laparoscopic LLS ${ }^{4,5,29-31}$ were of relatively small size and retrospective in nature. The addition of a prospective registry of patients who were not randomized owing to surgeon or patient preference was of value, reflecting daily practice in many units performing liver surgery ${ }^{22,23}$. 
In the present series of 67 LLLS and 24 OLLS procedures undertaken in conjunction with an ERAS programme, patients were functionally recovered after 3 days. Although median LOS tended to be slightly shorter in the laparoscopic groups, this difference was not significant, and the study was not powered to detect a difference in LOS. The clinical relevance of a difference of less than 1 day can be questioned. A delay to discharge after functional recovery was observed in all groups, and there is clearly the opportunity for a further reduction in LOS if discharge logistics could be optimized ${ }^{14,16}$. Minimization of this gap between recovery and discharge could reduce hospital costs.

Lessons learned from this trial could prove valuable for the design and execution of future surgical trials. Surgical RCTs are often difficult to undertake successfully and pose particular practical and methodological challenges ${ }^{32}$. Blinding is frequently difficult to perform, and care must be taken to choose an objectively measurable outcome ${ }^{33}$. The present study, however, confirmed the practicality of blinding patients through the use of large abdominal dressings ${ }^{34,35}$. Double-blinding could not be guaranteed as ward physicians read operation details accidentally. A proctoring surgeon assisted surgeons in their learning curve to ensure quality. Regression analyses in this study revealed no influence of individual surgeons on the primary outcome, supporting the view that this approach may overcome the surgical learning curve as a confounder ${ }^{32}$. The timing of the conduct of a surgical trial is also important ${ }^{32}$. When a new technique is introduced, there is a window of opportunity to conduct a trial. Once surgeons believe the new intervention is superior, randomization becomes difficult. This surgeon preference was the major cause of failure to recruit to this RCT. It is important that investigators ensure the presence of clinical equipoise. To assess this adequately, a feasibility study seems generally advisable to generate objective evidence that the main trial will not fail, at least due to a perceived lack of equipoise.

\section{+A: Collaborators}

The following are members of the ORANGE II Collaborative Group: T. Terkivatan (Erasmus Medical Centre, Rotterdam, The Netherlands), C. Verhoef (Erasmus Medical Centre, Rotterdam, The Netherlands), R. J. Porte (University Medical Centre Groningen, Groningen, The Netherlands), J. W. Haverman (University Medical Centre Groningen, Groningen, The Netherlands), O. R. Busch (Academic Medical 
Centre, Amsterdam, The Netherlands), M. A. Boermeester (Academic Medical Centre, Amsterdam, The Netherlands), M. G. Besselink (Academic Medical Centre, Amsterdam, The Netherlands), I. Q. Molenaar (University Medical Centre Utrecht, Utrecht, The Netherlands), I. H. M. Borel Rinkes (University Medical Centre Utrecht, Utrecht, The Netherlands), K. Bosscha (Jeroen Bosch Hospital, Den Bosch, The Netherlands), J. R. van der Vorst (Leiden University Medical Centre, Leiden, The Netherlands), J. W. D. de Waard (Westfriesgasthuis, Hoorn, The Netherlands), M. F. Gerhards (Onze Lieve Vrouw Gasthuis, Amsterdam, The Netherlands), G. A. Patijn (Isala, Zwolle, The Netherlands), M. Schmeding (University Hospital Aachen, Aachen, Germany; European HPB Centre Aachen-Maastricht), J. N. Primrose (University Hospital Southampton, Southampton, UK), M. Abu Hilal (University Hospital Southampton, Southampton, UK), I. Dagher (Hôpitaux Universitaires Paris Sud, Paris, France), A. Laurent (Hôpital Henri-Mondor, Paris, France), B. Topal (University Hospital Leuven, Leuven, Belgium), B. Edwin (Oslo University Hospital, Oslo, Norway), K. Lassen (Oslo University Hospital, Oslo, Norway) E. B. van Duyn (Medisch Spectrum Twente, Enschede, The Netherlands), A. W. Ambergen (Department of Methodology and Statistics, Maastricht University Medical Centre, Maastricht, The Netherlands) S. W. Olde Damink (Maastricht University Medical Centre, Maastricht, The Netherlands; European HPB Centre AachenMaastricht), M. H. Bemelmans (Maastricht University Medical Centre, Maastricht, The Netherlands; European HPB Centre Aachen-Maastricht).

\section{+A: Acknowledgements}

The authors acknowledge the HPB-surgical departments of Maastricht University Medical Centre (Maastricht, the Netherlands) and University Hospital Aachen (Aachen, Germany) and the Dutch Liver Surgery Group for their support. They also express their gratitude to J. N. Primrose for his continuing involvement in and constructive feedback to the ORANGE II study. The authors thank all patients for their participation, and local clinical personnel and research staff involved in recruitment and data collection. In addition, they thank DSMB members (M. A. Boermeester, I. H. M. Borel Rinkes and A. W. Ambergen) for their time, expert input and advice. 
The ORANGE II trial was funded predominantly by the Profiling Fund of Maastricht University Medical Centre, Maastricht, The Netherlands (number PF 298). The trial was also funded by the participating centres of the ORANGE II study group and supported by an unrestricted grant from Medtronic, Dublin, Ireland. Medtronic and other funders had no influence on the design of the trial, no access to trial data, and no influence on the communication of trial results.

Disclosure: The authors declare no conflict of interest.

\section{+A: References}

1. Buell JF, Cherqui D, Geller DA, et al. The international position on laparoscopic liver surgery: The Louisville Statement, 2008. Annals of surgery 2009; 250(5): 825-30.

2. Nguyen KT, Gamblin TC, Geller DA. World review of laparoscopic liver resection-2,804 patients. Annals of surgery 2009; 250(5): 831-41.

3. Reddy SK, Tsung A, Geller DA. Laparoscopic liver resection. World journal of surgery 2011; 35(7): 1478-86.

4. Abu Hilal M, McPhail MJ, Zeidan B, et al. Laparoscopic versus open left lateral hepatic sectionectomy: A comparative study. European journal of surgical oncology : the journal of the European Society of Surgical Oncology and the British Association of Surgical Oncology 2008; 34(12): 1285-8.

5. Aldrighetti L, Pulitano C, Catena M, et al. A prospective evaluation of laparoscopic versus open left lateral hepatic sectionectomy. Journal of gastrointestinal surgery : official journal of the Society for Surgery of the Alimentary Tract 2008; 12(3): 457-62.

6. Rao A, Rao G, Ahmed I. Laparoscopic left lateral liver resection should be a standard operation. Surg Endosc 2011; 25(5): 1603-10.

7. Hendry PO, van Dam RM, Bukkems SF, et al. Randomized clinical trial of laxatives and oral nutritional supplements within an enhanced recovery after surgery protocol following liver resection. The British journal of surgery 2010; 97(8): 1198-206.

8. Lin DX, Li X, Ye QW, Lin F, Li LL, Zhang QY. Implementation of a fast-track clinical pathway decreases postoperative length of stay and hospital charges for liver resection. Cell biochemistry and biophysics 2011; 61(2): 413-9.

9. MacKay G, O'Dwyer PJ. Early discharge following liver resection for colorectal metastases. Scott Med $J$ 2008; 53(2): 22-4.

10.van Dam RM, Hendry PO, Coolsen MM, et al. Initial experience with a multimodal enhanced recovery programme in patients undergoing liver resection. The British journal of surgery 2008; 95(8): 969-75.

11.Coolsen MM, Wong-Lun-Hing EM, van Dam RM, et al. A systematic review of outcomes in patients undergoing liver surgery in an enhanced recovery after surgery pathways. HPB : the official journal of the International Hepato Pancreato Biliary Association 2013; 15(4): 245-51.

12.Schultz NA, Larsen PN, Klarskov B, et al. Evaluation of a fast-track programme for patients undergoing liver resection. The British journal of surgery 2013; 100(1): 138-43.

13.Ni CY, Yang Y, Chang YQ, et al. Fast-track surgery improves postoperative recovery in patients undergoing partial hepatectomy for primary liver cancer: A prospective randomized controlled trial. European journal of surgical oncology : the journal of the European Society of Surgical Oncology and the British Association of Surgical Oncology 2013; 39(6): 542-7.

14.Stoot JH, van Dam RM, Busch OR, et al. The effect of a multimodal fast-track programme on outcomes in laparoscopic liver surgery: a multicentre pilot study. HPB : the official journal of the International Hepato Pancreato Biliary Association 2009; 11(2): 140-4. 
15.Jones C, Kelliher L, Dickinson M, et al. Randomized clinical trial on enhanced recovery versus standard care following open liver resection. The British journal of surgery 2013; 100(8): 1015-24.

16.Maessen J, Dejong CH, Hausel J, et al. A protocol is not enough to implement an enhanced recovery programme for colorectal resection. The British journal of surgery 2007; 94(2): 224-31.

17.van Dam RM, Wong-Lun-Hing EM, van Breukelen GJ, et al. Open versus laparoscopic left lateral hepatic sectionectomy within an enhanced recovery ERAS(R) programme (ORANGE II-trial): study protocol for a randomised controlled trial. Trials 2012; 13: 54.

18.Brewin CR, Bradley C. Patient preferences and randomised clinical trials. Bmj 1989; 299(6694): 3135.

19.Gore SM. The consumer principle of randomisation. Lancet 1994; 343(8888): 58.

20.Paice JA, Cohen FL. Validity of a verbally administered numeric rating scale to measure cancer pain intensity. Cancer nursing 1997; 20(2): 88-93.

21.McGuire DB. Comprehensive and multidimensional assessment and measurement of pain. Journal of pain and symptom management 1992; 7(5): 312-9.

22.Krebs EE, Carey TS, Weinberger M. Accuracy of the pain numeric rating scale as a screening test in primary care. Journal of general internal medicine 2007; 22(10): 1453-8.

23.Suurmeijer TP, Doeglas DM, Moum T, et al. The Groningen Activity Restriction Scale for measuring disability: its utility in international comparisons. American journal of public health 1994; 84(8): 1270-3.

24.Dindo D, Demartines N, Clavien PA. Classification of surgical complications: a new proposal with evaluation in a cohort of 6336 patients and results of a survey. Annals of surgery 2004; 240(2): 20513.

25.van den Broek MA, van Dam RM, van Breukelen GJ, et al. Development of a composite endpoint for randomized controlled trials in liver surgery. The British journal of surgery 2011; 98(8): 1138-45.

26.Dana-Farber / Harvard Cancer Center, Accrual and Scientific Progress by the Scientific Review Committee, SOP \#: COM-102. Available from:

http://www.dfhcc.harvard.edu/fileadmin/DFHCC_Admin/Clinical_Trials/CRO/Policies_and_Procedur es-SOPs/COM-102_Version_10.pdf?PHPSESSID=645544e7b583b68fe5338434cfb89bc5 (accessed 12-05-2014).

27.Wong-Lun-Hing EM, Lodewick TM, Stoot JH, et al. A survey in the hepatopancreatobiliary community on ways to enhance patient recovery. $H P B$ : the official journal of the International Hepato Pancreato Biliary Association 2012; 14(12): 818-27.

28.Wakabayashi G, Cherqui D, Geller DA, et al. Recommendations for laparoscopic liver resection: a report from the second international consensus conference held in Morioka. Annals of surgery 2015; 261(4): 619-29.

29.Carswell KA, Sagias FG, Murgatroyd B, Rela M, Heaton N, Patel AG. Laparoscopic versus open left lateral segmentectomy. BMC surgery 2009; 9: 14.

30.Endo Y, Ohta M, Sasaki A, et al. A comparative study of the long-term outcomes after laparoscopyassisted and open left lateral hepatectomy for hepatocellular carcinoma. Surgical laparoscopy, endoscopy \& percutaneous techniques 2009; 19(5): e171-4.

31.Tang CN, Tai CK, Ha JP, Siu WT, Tsui KK, Li MK. Laparoscopy versus open left lateral segmentectomy for recurrent pyogenic cholangitis. Surg Endosc 2005; 19(9): 1232-6.

32.Cook JA. The challenges faced in the design, conduct and analysis of surgical randomised controlled trials. Trials 2009; 10: 9.

33.Lilford R, Braunholtz D, Harris J, Gill T. Trials in surgery. The British journal of surgery 2004; 91(1): 6-16.

34.Katkhouda N, Mason RJ, Towfigh S, Gevorgyan A, Essani R. Laparoscopic versus open appendectomy: a prospective randomized double-blind study. Annals of surgery 2005; 242(3): 439-48; discussion 48-50.

35.Seiler CM, Deckert A, Diener MK, et al. Midline versus transverse incision in major abdominal surgery: a randomized, double-blind equivalence trial (POVATI: ISRCTN60734227). Annals of surgery 2009; 249(6): 913-20. 
Fig. 2 CONSORT diagram for the trial. LLS, left lateral sectionectomy; OLLS, open left lateral sectionectomy; LLLS, laparoscopic left lateral sectionectomy 
Table 1 Perioperative care according to the enhanced recovery after liver surgery programme

\begin{tabular}{|c|c|}
\hline & Daily guideline \\
\hline \multirow[t]{3}{*}{ Day before surgery } & Normal oral nutrition up to $6 \mathrm{~h}$ before surgery \\
\hline & No preanaesthetic medication \\
\hline & Laboratory tests \\
\hline \multirow[t]{10}{*}{ Day of surgery } & Carbohydrate drinks up to $2 \mathrm{~h}$ before surgery \\
\hline & Mid-thoracic epidural analgesia (local anaesthetic + low-dose opioid) \\
\hline & Short-acting i.v. anaesthetic agent \\
\hline & Preferably no nasogastric drainage, but when used remove after surgery \\
\hline & Use warm i.v. fluids, and upper and lower body air-warming device \\
\hline & Avoid excessive i.v. fluids \\
\hline & CVP during transection $<5 \mathrm{mmHg}$ \\
\hline & No routine drainage of peritoneal cavity \\
\hline & Patient sent to recovery ward \\
\hline & Restart oral intake of water/nutrition \\
\hline \multirow[t]{10}{*}{ POD 1} & Patient sent to surgical ward \\
\hline & Patient mobilizes a minimum of four times a day \\
\hline & Discontinuation of i.v. fluids \\
\hline & Patient drinks at least 1.5 litres \\
\hline & Normal diet \\
\hline & Continue portable epidural analgesia (local anaesthetic-low-dose opioid) or PCA \\
\hline & Remove urinary catheter \\
\hline & $1000 \mathrm{mg}$ paracetamol 6-hourly \\
\hline & Start laxatives \\
\hline & Laboratory tests \\
\hline \multirow[t]{6}{*}{ POD 2} & Continue portable epidural analgesia or PCA \\
\hline & Stop low-dose opioids \\
\hline & Continue mobilization a minimum of four times daily \\
\hline & 1000 mg paracetamol 6-hourly \\
\hline & Normal diet \\
\hline & Laxatives \\
\hline \multirow[t]{7}{*}{ POD 3} & Stop epidural analgesia or PCA \\
\hline & Start NSAIDs \\
\hline & Continue mobilization \\
\hline & Normal diet \\
\hline & Laboratory tests \\
\hline & Check discharge criteria \\
\hline & Outpatient appointment made for POD 10,11 or 12 \\
\hline \multirow[t]{3}{*}{ POD 4} & Check discharge criteria \\
\hline & Patient given mobile phone number of HPB surgeon on duty \\
\hline & Discharge \\
\hline
\end{tabular}

i.v., Intravenous; POD, postoperative day; CVP, central venous pressure; PCA, patient-controlled analgesia; NSAID, non-steroidal antiinflammatory drug; HPB, hepatopancreatobiliary. 
Table 2 Patient demographics

\begin{tabular}{|c|c|c|c|c|c|c|}
\hline & \multicolumn{3}{|c|}{ RCT } & \multicolumn{3}{|c|}{ Registry } \\
\hline & $\operatorname{OLLS}(n=11)$ & $\operatorname{LLLS}(n=13)$ & $P \#$ & ONR $(n=13)$ & $\operatorname{LNR}(n=54)$ & $P \#$ \\
\hline Age (years)* & $58(52-70)$ 材 & 67 (55-73)杮 & $0.361^{* *}$ & $53(46-64)$ & $63(45-72)$ & $0.219^{* *}$ \\
\hline Sex ratio $(M: F)$ & $5: 6$ & $9: 4$ & 0.408 & $8: 5$ & $26: 28$ & $0.386 \dagger \dagger$ \\
\hline BMI $\left(\mathrm{kg} / \mathrm{m}^{2}\right)^{*}$ & 28.7 (25.5-33.9)杮 & 27.1 (25.3-28.5)㧊 & $0.361^{* *}$ & $23.5(22.0-27.2)$ & $24.7(22.5-29.2)$ & $0.306^{* *}$ \\
\hline ASA fitness grade & & & $0.252^{* *}$ & & & $0.851^{* *}$ \\
\hline I & $3(27)$ & $1(8)$ & & $4(31)$ & $17(31)$ & \\
\hline II & $7(64)$ & $9(69)$ & & $8(62)$ & $30(56)$ & \\
\hline III & $1(9)$ & $3(23)$ & & $1(8)$ & $7(13)$ & \\
\hline Indication for surgery & & & 0.182 & & & $0.267 \dagger \dagger$ \\
\hline $\begin{array}{l}\text { Colorectal } \\
\text { metastasis }\end{array}$ & $6(55)$ & $11(85)$ & & $7(54)$ & $20(37)$ & \\
\hline Other & $5(45) \dagger$ & $2(15) \ddagger$ & & $6(46) \S$ & 34 (63)ף & \\
\hline $\begin{array}{l}\text { Previous abdominal } \\
\text { surgery }\end{array}$ & $9(82)$ & $11(85)$ & 1.000 & $9(69)$ & $24(44)$ & $0.048 \dagger \dagger$ \\
\hline $\begin{array}{l}\text { Preoperative } \\
\text { chemotherapy }\end{array}$ & $3(27)$ & $9(69)$ & 0.100 & $3(23)$ & $14(26)$ & 1.000 \\
\hline
\end{tabular}

Values in parentheses are percentages unless indicated otherwise; *values are median (i.q.r.). OLLS, open left lateral sectionectomy; LLLS, laparoscopic left lateral sectionectomy; ONR, open non-randomized left lateral sectionectomy; LNR, laparoscopic non-randomized left lateral sectionectomy. †Adenoma (2), hepatocellular carcinoma (HCC) (1), haemangioma (1), focal nodular hyperplasia (FNH) (1); ¥HCC (1), multilocular biliary cyst (1); §HCC (3), metastatic melanoma (1), liver abscess (1), metastatic breast cancer (1); ๆHCC (3), haemangioma (4), adenoma (5), FNH (7), inflammatory lesions (2), echinococcosis (1), hepatic cyst (1), haemangioma (1). \#Fisher's exact test, except **MannWhitney $U$ test and $\dagger \dagger \chi^{2}$ test (all two-tailed). 抹ariable with normal distribution: Shapiro-Wilk $P>0.050$; Mann-Whitney $U$ test results are shown, but $t$ test leads to the same conclusion. $\alpha=0.01$ for all baseline variables to correct for multiple testing. 
Table 3 Surgical outcomes

\begin{tabular}{|c|c|c|c|c|c|c|}
\hline & \multicolumn{3}{|c|}{ RCT } & \multicolumn{3}{|c|}{ Registry } \\
\hline & OLLS $(n=11)$ & LLLS $(n=13)$ & $P$ 材 & ONR $(n=13)$ & LNR $(n=54)$ & $P$ 中市 \\
\hline \multicolumn{7}{|l|}{ Type of liver resection } \\
\hline LLS & $10(91)$ & $11(85)$ & & $10(77)$ & $46(85)$ & \\
\hline LLS + wedge & $1(9) \dagger$ & $2(15) \ddagger$ & & $3(23) \S$ & $2(4) 9$ & \\
\hline Segment III & $0(0)$ & $0(0)$ & & $0(0)$ & $1(2)$ & \\
\hline Conversion to open surgery & & $0(0)$ & & & 5 (9)\# & \\
\hline \multicolumn{7}{|l|}{ Additional procedures } \\
\hline Cholecystectomy & $1(9)$ & $1(8)$ & & $1(8)$ & $3(6)$ & \\
\hline Lymph node dissection & $0(0)$ & $0(0)$ & & $1(8)^{* *}$ & $1(2) \dagger \dagger$ & \\
\hline Hernia correction & $0(0)$ & $0(0)$ & & $0(0)$ & $2(4)$ & \\
\hline Other & 1 (9)杮 & $1(8) \S \S$ & & $0(0)$ & $2(4) \boldsymbol{\top}$ & \\
\hline Duration of surgery (min)* & $110(92-125)$ ๆศศ & 156 (112-176)ளศף & $0.023 \S \S \S$ & 206 (118-255)ศाศ & 148 (118-202)ตाศ & $0.082 \S \S \S$ \\
\hline Blood loss (ml)* & $100(100-350)$ & $50(18-200)$ & $0.063 \S \S \S$ & $250(225-300)$ & $200(100-300)$ & $0.191 \S \S \S$ \\
\hline Vascular control & 2 (18)\#\# & $0(0)$ & 0.199 & $2(15)^{* * *}$ & $5(9) \dagger \dagger \dagger$ & 0.614 \\
\hline Placement of abdominal drain & $0(0)$ & $1(8)$ & 1.000 & $5(38)$ & $12(22)$ & 0.289 \\
\hline
\end{tabular}

Values in parentheses are percentages unless indicated otherwise; *values are median (i.q.r.). OLLS, open left lateral sectionectomy; LLLS, laparoscopic left lateral sectionectomy; ONR, open non-randomized left lateral sectionectomy; LNR, laparoscopic non-randomized left lateral sectionectomy; LLS, left lateral sectionectomy. †Segment IVB; łsegment IVB (2); §segments V, IVB and VIII; ๆsegment VI and segment IVB (2). \#Reason for conversion: tumour to close to vascular structures (1), adhesions (1), bleeding from aberrant left hepatic artery (1), size of lesion (1), infiltration of diaphragm (1). ${ }^{* *}$ Hepatoduodenal ligament + omentum minus + left gastric artery + coeliac artery + gastroduodenal artery.

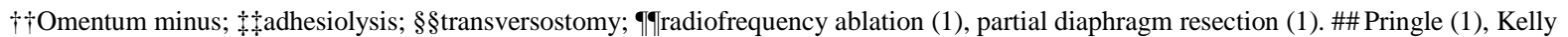

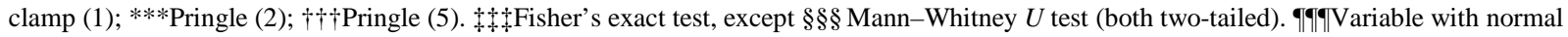
distribution: Shapiro-Wilk $P>0.050$; Mann-Whitney $U$ test results are shown, but $t$ test leads to the same conclusion. $\alpha=0.01$ for all secondary outcomes to correct for multiple testing. 
Table 4 Functional recovery and length of hospital stay

\begin{tabular}{|c|c|c|c|c|c|c|}
\hline & \multicolumn{3}{|c|}{ RCT } & \multicolumn{3}{|c|}{ Registry } \\
\hline & OLLS $(n=11)$ & LLLS $(n=13)$ & $P \dagger$ & ONR $(n=13)$ & LNR $(n=54)$ & $P \dagger$ \\
\hline Functional recovery (days) & $3(3-5)$ & $3(3-3)$ & 0.284 & $3(3-3)$ & $3(3-4)$ & 0.529 \\
\hline $\begin{array}{l}\text { Adequate pain control with oral analgesia } \\
\text { only }\end{array}$ & $3(2-3)$ & $3(2-3)$ & 0.539 & $3(3-4)$ & $2(2-3)$ & 0.017 \\
\hline Independent mobility or preoperative level & $3(3-4)$ & $3(2-3)$ & 0.071 & $3(3-4)$ & $3(2-3)$ & 0.240 \\
\hline No intravenous fluid & $2.5(2-3) \S$ & $2(1-3) \S$ & 0.273 & $2(1-4)$ & $2(1-2)$ & 0.308 \\
\hline Tolerance of solid food & $1(1-1)$ & $1(1-1)$ & 0.738 & $2(1-2)$ & $1(1-1)$ & 0.002 \\
\hline Normal or decreasing serum bilirubin level & $2.5(1-3)$ & $1(1-3)$ & 0.232 & $0(0-1)$ & $1(0-2)$ & 0.161 \\
\hline \multicolumn{7}{|l|}{ Postoperative milestones (days) } \\
\hline Free oral fluids & $0(0-1)$ & $0(0-0)$ & 0.563 & $1(0-1)$ & $1(0-1)$ & 0.202 \\
\hline Removal of indwelling urinary catheter & $3(2-3)$ & $2 \cdot 5(1-3)$ & 0.140 & $3(3-6)$ & $2(1-3)$ & 0.031 \\
\hline First flatus & $1(1-2)$ & $1(1-2)$ & 0.446 & $2(1-3)$ & $2(1-2)$ & 0.076 \\
\hline First stool & $3(2-4) \S$ & $2(2-3) \S$ & 0.307 & $3(3-4) \S$ & $2(2-3) \S$ & 0.138 \\
\hline LOS (days) & $4.5(4-6)$ & $4(3-5)$ & 0.049 & $5(4-7)$ & $4(3-5)$ & 0.064 \\
\hline Difference (LOS - functional recovery) (days) & $1(0-3)$ & $1(1-2)$ & 0.832 & $2(1-3)$ & $1(0-2)$ & 0.042 \\
\hline Delay in discharge* ${ }^{*}$ & 8 of $10(80)$ & $9(69)$ & $1.000 \ddagger$ & $11(85)$ & $23(43)$ & $0.090 \ddagger$ \\
\hline \multicolumn{7}{|l|}{ Reasons for delay in discharge* } \\
\hline Logistical & 2 of $10(20)$ & $5(38)$ & & $6(46)$ & $11(20)$ & \\
\hline Medical & 3 of $10(30)$ & $1(8)$ & & $2(15)$ & $5(9)$ & \\
\hline Patient preference & 2 of $10(20)$ & $2(15)$ & & $0(0)$ & $2(4)$ & \\
\hline Unknown & 1 of $10(10)$ & $1(8)$ & & $3(23)$ & $15(28)$ & \\
\hline
\end{tabular}

Values are median (i.q.r.) unless indicated otherwise; *values in parentheses are percentages. †One patient died in hospital and was never discharged. OLLS, open left lateral sectionectomy; LLLS, laparoscopic left lateral sectionectomy; ONR, open non-randomized left lateral sectionectomy; LNR, laparoscopic non-randomized left lateral sectionectomy; LOS, length of hospital stay. OLLS group: reason for delay in discharge $\mathrm{n}=10$, due to one mortality. $†$ Mann-Whitney $U$ test, except †Fisher’s exact test (both two-tailed). §Variable with normal distribution: Shapiro-Wilk $P>0.050$; Mann-Whitney $U$ test results are shown, but $t$ test leads to the same conclusion. $\alpha=0.05$ for primary outcome, and $\alpha=0.01$ for all secondary outcomes to correct for multiple testing. 
Table 5 Complications

\begin{tabular}{|l|c|c|c|c|c|c|}
\hline \multirow{2}{*}{} & \multicolumn{3}{|c|}{ RCT } & \multicolumn{3}{c|}{ Registry } \\
\cline { 2 - 7 } & $\begin{array}{c}\text { OLLS } \\
(n=11)\end{array}$ & $\begin{array}{c}\text { LLLS } \\
(n=13)\end{array}$ & $P \ddagger$ & $\begin{array}{c}\text { ONR } \\
(n=13)\end{array}$ & $\begin{array}{c}\text { LNR } \\
(n=54)\end{array}$ & $P \ddagger$ \\
\hline Overall morbidity & $4(36)$ & $1(8)$ & 0.141 & $2(15)$ & $7(13)$ & 1.000 \\
\hline Clavien-Dindo grade ${ }^{23}$ & & & & & & \\
\hline No morbidity & $7(64)$ & $12(92)$ & & $11(85)$ & $47(87)$ & \\
\hline I & $1(9)$ & $0(0)$ & & $0(0)$ & $0(0)$ & \\
\hline II & $2(18)$ & $0(0)$ & & $1(8)$ & $5(9)$ & \\
\hline III & $0(0)$ & $1(8)$ & & $1(8)$ & $1(2)$ & \\
\hline IV & $0(0)$ & $0(0)$ & & $0(0)$ & $1(2)$ & \\
\hline V (30-day mortality) & $1(9)$ & $0(0)$ & & $0(0)$ & $0(0)$ & \\
\hline Major morbidity $\geq$ grade III) & $1(9)$ & $1(8)$ & 1.000 & $1(8)$ & $2(4)$ & 0.482 \\
\hline Composite endpoint* & $1(9)$ & $0(0)$ & 0.458 & $1(8)$ & $2(4)$ & 0.482 \\
\hline Readmission in $<30$ days & $0(0)$ & $0(0)$ & & $0(0)$ & $1(2)$ & 1.000 \\
\hline Complications & & & & & & \\
\hline Wound infection & $1(9)$ & $1(8)$ & & $0(0)$ & $1(2)$ & \\
\hline Pneumonia & $0(0)$ & $0(0)$ & & $0(0)$ & $2(4)$ & \\
\hline Intra-abdominal haemorrhage & $0(0)$ & $0(0)$ & & $1(8)$ & $0(0)$ & \\
\hline Intra-abdominal abscess & $0(0)$ & $0(0)$ & & $0(0)$ & $1(2)$ & \\
\hline Postresectional liver failure & $0(0)$ & $0(0)$ & & $0(0)$ & $1(2)$ & \\
\hline Pulmonary embolism & $1(9)$ & $0(0)$ & & $0(0)$ & $0(0)$ & \\
\hline Cardiac arrest & $1(9)$ & $0(0)$ & & $0(0)$ & $0(0)$ & \\
\hline Other† & $2(18)$ & $0(0)$ & & $2(15)$ & $3(6)$ & \\
\hline
\end{tabular}

Values in parentheses are percentages. OLLS, open left lateral sectionectomy; LLLS, laparoscopic left lateral sectionectomy; ONR, open non-randomized left lateral sectionectomy; LNR, laparoscopic non-randomized left lateral sectionectomy. *Ascites, postresectional liver failure, bile leakage, intra-abdominal haemorrhage, intraabdominal abscess and operative mortality. †Persistent pain (2), hypertension (2), infected epidural insertion site (1), urinary tract infection (1), dyspnoea of unknown origin (1). ‡Fisher’s exact test (two-tailed). $\alpha=0.01$ for all secondary outcomes to correct for multiple testing. 
Table 6 Overall compliance to elements of the enhanced recovery after surgery protocol per study group

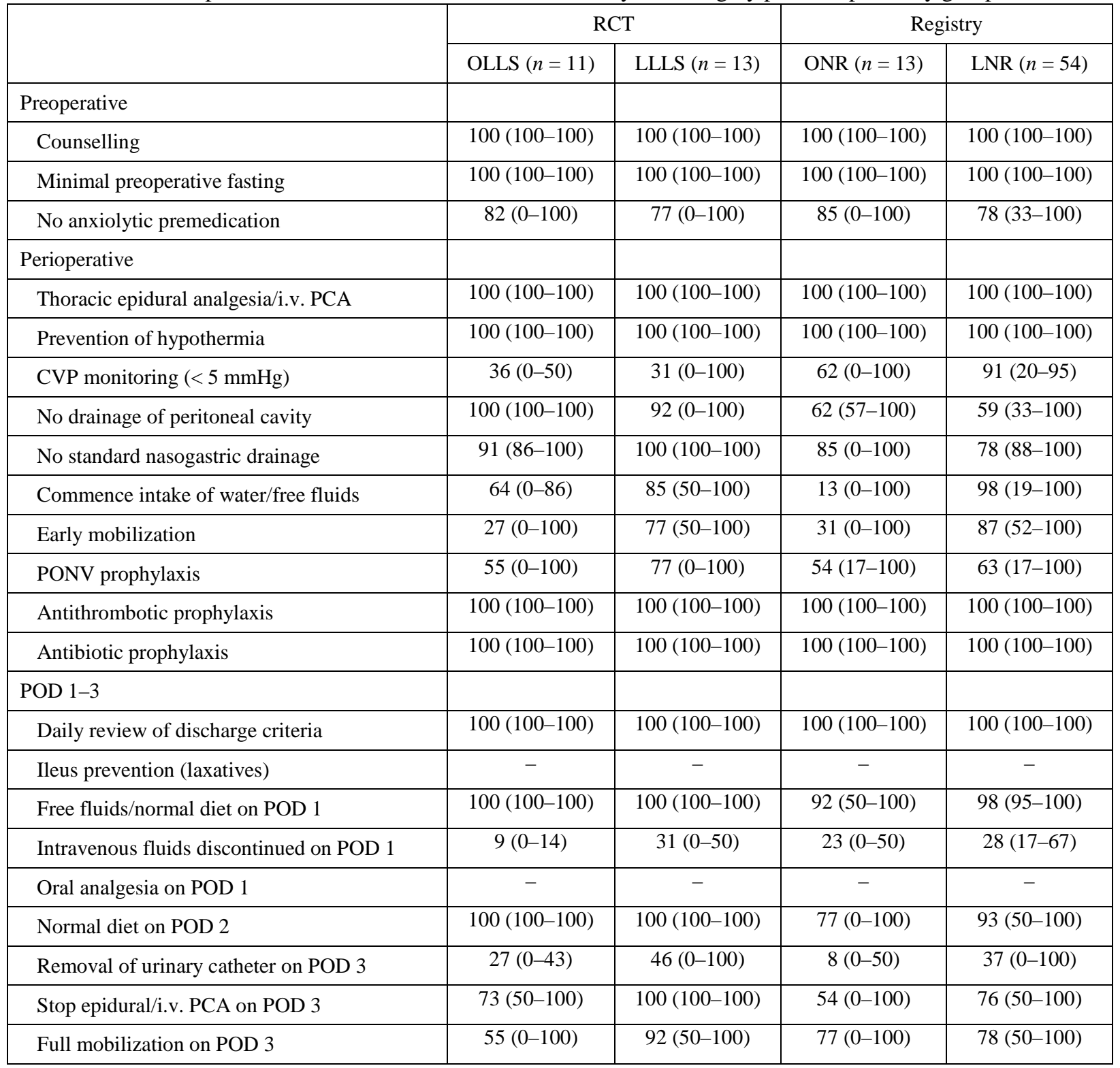

Values are percentages with between-centre ranges in parentheses. OLLS, open left lateral sectionectomy; LLLS, laparoscopic left lateral sectionectomy; ONR, open non-randomized left lateral sectionectomy; LNR, laparoscopic non-randomized left lateral sectionectomy. i.v., Intravenous; PCA, patient-controlled analgesia; CVP, central venous pressure; PONV, postoperative nausea and vomiting; POD, postoperative day. 